\title{
Safe laparoscopic surgery in median arcuate ligament syndrome
}

\author{
Ulaş Aday ${ }^{1}$, Abdullah Böyük², Barış Gültürk², Mehmet Buğra Bozan² \\ ${ }^{1}$ Department of Gastroenterological Surgery, University of Health Sciences, Elazıg Training and Research Hospital, Elazıg, Turkey \\ ${ }^{2}$ Department of Surgery, University of Health Sciences, Elazıg Training and Research Hospital, Elazıg, Turkey
}

Videosurgery Miniinv 2018; 13 (4): 539-541

DOI: https://doi.org/10.5114/wiitm.2018.76116

\begin{abstract}
The median arcuate ligament syndrome is a rare clinical condition with no standardized method of laparoscopic treatment. Exposure of the aorto-celiac axis might be considered as the most challenging part of the surgical procedure. It is important to secure total release while enabling adequate vision as the compressing musculofibrous ligament is located in the deepest part of the aorto-celiac hiatus. A 29-year-old male patient presenting with recurrent abdominal pain and diagnosed with the median arcuate ligament syndrome underwent laparoscopic surgery. The patient was discharged without problems on the fourth day after the surgery. In this case report we present a maneuver that enables easy and safe exposure of the celiac trunk.
\end{abstract}

Key words: median arcuate ligament syndrome, laparoscopic surgery, operation technique.

\section{Introduction}

The median arcuate ligament syndrome (MALS), also known as the celiac artery compression syndrome, is a rare clinical condition that is characterized by chronic abdominal pain triggered by meals, nausea and/or vomiting, and weight loss. The syndrome results from the median arcuate ligament (MAL), the continuation of the diaphragm, causing extrinsic compression on the celiac artery (CA) at the point where it crosses the aorta at a lower level and thus narrowing the CA [1]. The dissection of the compressing musculofibrous structure in order to release the compression on the CA is a plausible method of treatment of MALS. It has also been concluded that laparoscopic surgery may safely be performed in the treatment of MALS [2]. There are various techniques used for the laparoscopic approach. Surgeons switch to open surgery in $13 \%$ to $27 \%$ of cases due to either inadequate visualization of the anatomy or excessive hemorrhages [3, 4]. Treatment of MALS is aimed at restoring normal blood flow in the CA and eliminating neural irritation produced by the celiac ganglion fibers. The exposure of the aorto-celiac axis represents the most challenging part of the surgical procedure. It is important to secure total release while enabling adequate vision as the compressing musculofibrous ligament is located in the deepest part of the aorto-celiac hiatus $[2,5,6]$.

This case report presents a method that enables exposure of the MAL while minimizing vascular injury.

\section{Case report}

A 29-year-old male patient was admitted to our clinic complaining of severe abdominal pain after meals for one month. The patient had no history of chronic disease or drug use. He was a smoker and had involuntarily lost $22 \mathrm{~kg}$ within the past month. His physical examination was normal. The results of his laboratory tests, abdominal ultrasonic evaluation, and gastroscopy were also within normal limits. The patient's angiographic computed tomography

\section{Address for correspondence}

Ulaş Aday, Department of Gastroenterological Surgery, University of Health Sciences, Elazıg Training and Research Hospital, Elazıg,

Turkey, phone: +90 5302933895, e-mail: ulasaday@gmail.com 
and magnetic resonance imaging, however, showed findings consistent with MALS (Photo 1). The patient then was informed about the surgical procedure and his written consent was obtained. The surgical procedure was completed in 105 min without compli-

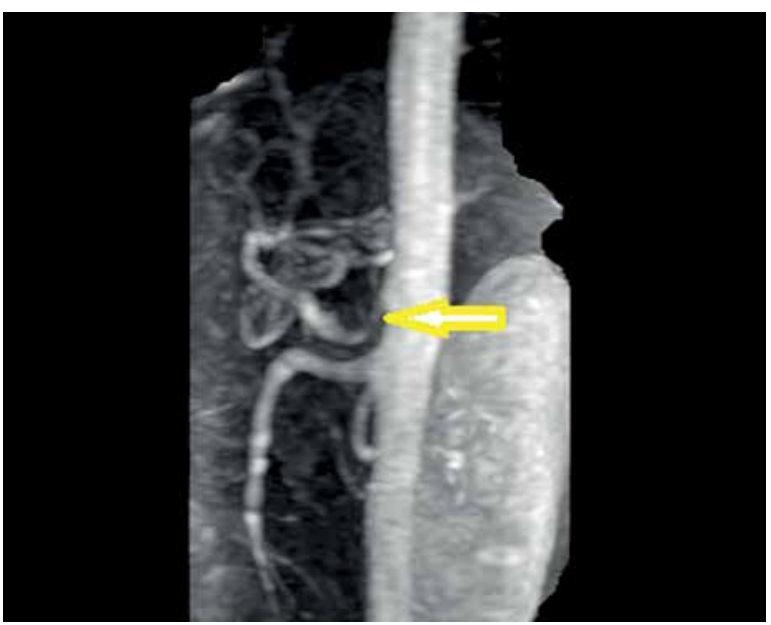

Photo 1. Abdominal angiographic magnetic resonance image of narrowness in the celiac artery
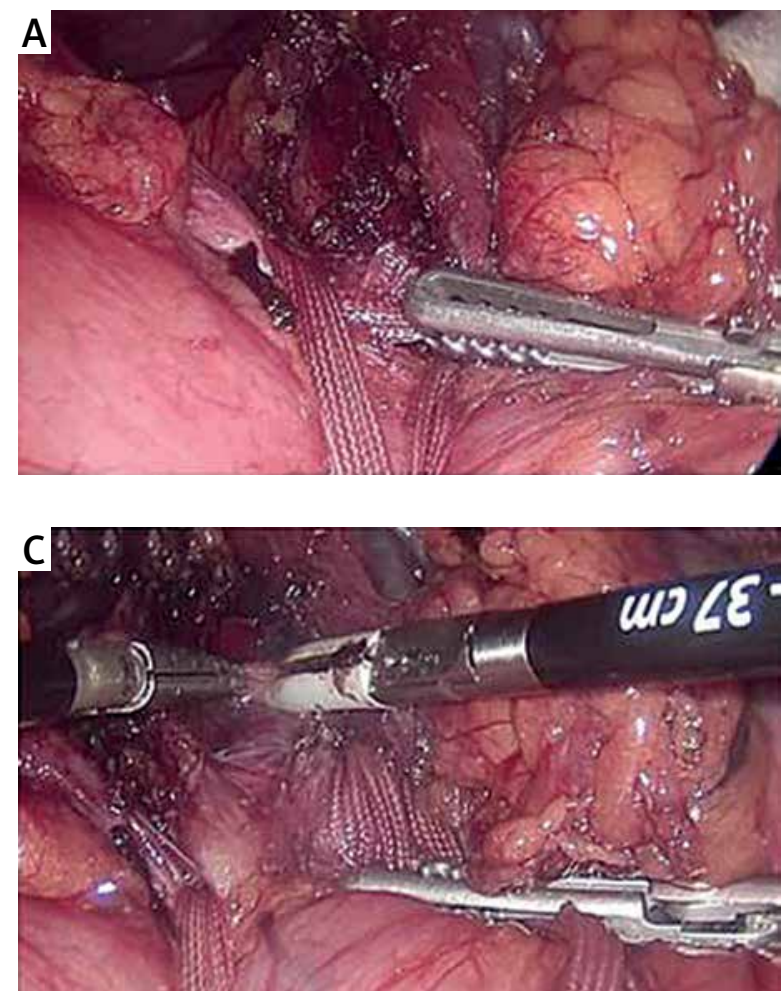

cations. The patient was discharged on the $4^{\text {th }}$ day after surgery without problems. He is now in the $6^{\text {th }}$ month of follow-up and is non-symptomatic.

\section{Operation technique}

Under general anesthesia, the patient was placed in a supine position in the $30^{\circ}$ reverse Trendelenburg position with legs apart and the surgeon stood between the patient's legs. A camera port was inserted on the midline at about $5 \mathrm{~cm}$ above the umbilicus. A 5-mm trocar was inserted into the right subcostal area, while a $10-\mathrm{mm}$ trocar medially and two $5-\mathrm{mm}$ trocars laterally were inserted into the left subcostal area under direct vision. An automatic retractor was placed under the xiphoid in order to elevate the left lobe of the liver. The gastrohepatic ligament was opened up following abdominal exploration. The left gastric artery (LGA) and arteria hepatica communis (AHC) were isolated and tape was placed around them. The right crus was dissected with an energy device and the anterior surface of the aorta was exposed. The LGA and AHC were held medially and lat-
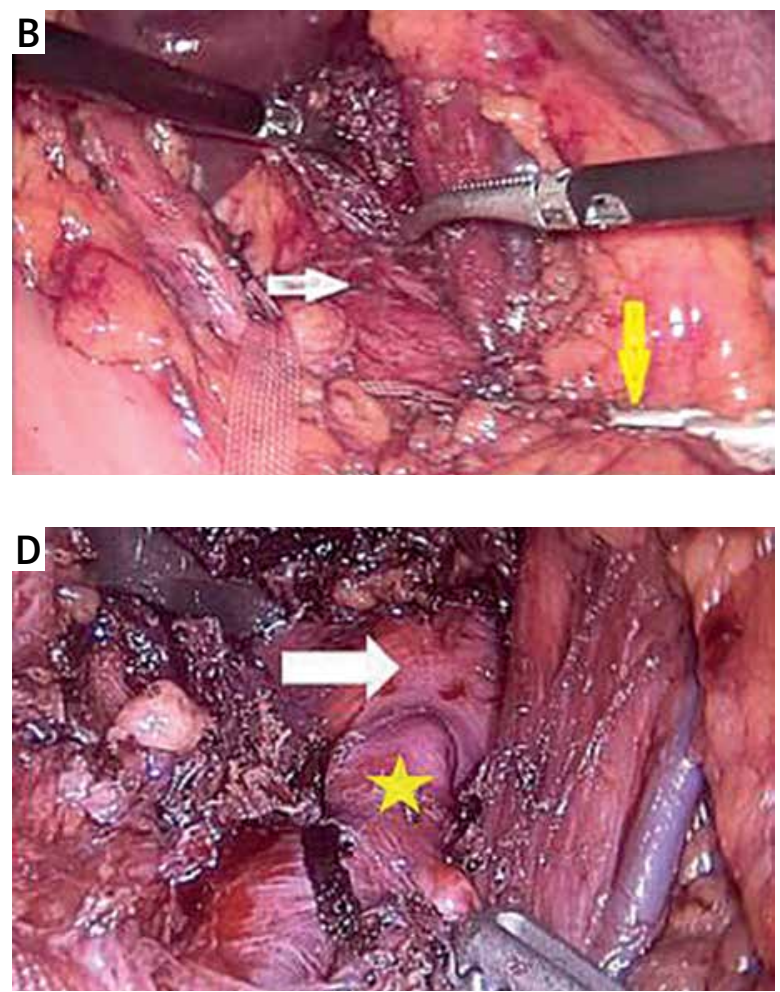

Photo 2. A - The hepatic and left gastric arteries were pulled with tape, B - when the vascular tape is fully pulled down and on the left side (yellow arrow) the bands on the celiac artery are clearly visible (white arrow), $\mathbf{C}$ - musculo-fibrotic band is cut by energy device, $\mathbf{D}$ - The aorta (marked with a white arrow) and the celiac artery (marked with asterisks) were completely released 
erally with tape and an endograsper/endoclinch and traction was applied towards the caudal and slightly to the left side of the patient. Through this maneuver, the fibrotic structures on the CA were visualized and they were divided by the energy device. All the potential fibrotic bands up to the part where the CA exited the aorta were released (Photo 2 ).

\section{Discussion}

Median arcuate ligament syndrome is a rare problem that surgeons encounter in surgical practice. Anatomically, the MAL is a musculofibrous structure connecting the two diaphragmatic crura from the anterior at the aortic hiatus level [6]. When sufficient blood flow cannot be achieved after meals due to the compression of the CA, patients develop symptoms. Postprandial epigastric ischemic abdominal pain and weight loss are frequently observed. It has been recommended that the musculofibrous structures and MAL causing the compression should be removed in symptomatic patients $[1,4,5]$. In 1965, Dunbar reported division of the MAL in 13 patients, with good results [7]. Today, laparoscopic surgery is being used at increasing rates. The laparoscopic method has advantages such as avoiding median laparotomy, less postoperative pain, short duration of hospitalization, early oral intake initiation, and better vision during the surgery. Since Roayaie et al. [8] first defined laparoscopic CA release, open surgery rates have dropped below laparoscopy rates over the years $[2,5,8]$. In the literature, results from the limited number of robotic assisted procedures are reported to be similar to those from laparoscopic surgery [9]. There is no standardized and commonly accepted method for laparoscopic techniques. All fibrotic bands should be divided by surgery, leaving the celiac trunk completely free circumferentially. Surgeons need to overcome two problems when dissecting the bands causing compression. The first one is to achieve good vision by exposing the anatomy. The second one is to complete the procedure without vascular injuries [2, 4-6]. Theoretically the tightest ligament that causes compression on the CA is the deepest one. Therefore, all the tight fibrous bands should be dissected to the point where the aorta is exposed through denudation of the CA wall. We believe that the maneuver we have described significantly enables surgical visualization of the celiac trunk without hemorrhage. The placement of tape around the CHA and the LGA both from the lateral side and the midpoint of both arteries towards the caudal and the left side of the patient facilitates the surgery. Moreover, this maneuver obviates the need of a tape around the esophagus for suspension. It is important to avoid extreme and prolonged traction of the CHA while performing this maneuver in order to preserve hepatic arterial flow.

\section{Conclusions}

Laparoscopic surgery is safe when appropriate maneuvering is performed, and the results are satisfactory. The maneuver we proposed not only is a safe method in the surgical treatment of the MALS but also facilitates the surgery.

\section{Conflict of interest}

The authors declare no conflict of interest.

\section{References}

1. Baccari P, Civilini E, Dordoni L, et al. Celiac artery compression syndrome managed by laparoscopy. J Vasc Surg 2009; 50: 134-9.

2. Cienfuegos JA, Estevez MG, Ruiz-Canela M, et al. Laparoscopic treatment of median arcuate ligament syndrome: analysis of long-term outcomes and predictive factors. J Gastrointest Surg 2018; 22: 713-21.

3. Berard X, Cau J, Déglise S, et al. Laparoscopic surgery for coeliac artery compression syndrome: current management and technical aspects. Eur J Vasc Endovasc Surg 2012; 43: 38-42.

4. Roseborough GS. Laparoscopic management of celiac artery compression syndrome. J Vasc Surg 2009; 50: 124-33.

5. Duffy AJ, Panait L, Eisenberg D, et al. Management of median arcuate ligament syndrome: a new paradigm. Ann Vasc Surg 2009; 23: 778-84.

6. Kim EN, Lamb K, Relles D, et al. Median arcuate ligament syndrome - review of this rare disease. JAMA Surg 2016; 151: 471-7.

7. Dunbar JD, Molnar W, Beman FF, Marable SA. Compression of the celiac trunk and abdominal angina. Am J Roentgenol Radium Ther Nucl Med 1965; 95: 731-4.

8. Roayaie S, Jossart G, Gitlitz D, et al. Laparoscopic release of celiac artery compression syndrome facilitated by laparoscopic ultrasound scanning to confirm restoration offlow. J Vasc Surg 2000; 32: 814-7.

9. Do MV, Smith TA, Bazan HA, et al. Laparoscopic versus robotassisted surgery for median arcuate ligament syndrome. Surg Endosc 2013; 27: 4060-6.

Received: 7.04.2018, accepted: 9.05.2018. 